\title{
Approaches for Improving Health Equity: The Effect of The Gainesville Community Resource Paramedic Program on Reducing Emergency Department Utilization
}

Andrew S Cistola ( $\sim$ andrewcistola@ufl.edu )

University of Florida https://orcid.org/0000-0002-0427-1567

Ariella N Bak

City of Gainesville

Laura Guyer

University of Florida

Austin Reed

University of Florida College of Medicine

Ben Rooks

University of Florida College of Medicine

Lisa Chacko

University of Florida College of Medicine

\section{Research}

Keywords: ED, CRP, PCP, Paramedic

Posted Date: October 8th, 2020

DOI: https://doi.org/10.21203/rs.3.rs-87078/v1

License: (c) (i) This work is licensed under a Creative Commons Attribution 4.0 International License.

Read Full License 


\section{Abstract}

Background. The U.S. healthcare system has consistently struggled with inefficiencies in Emergency Department (ED) usage (Enard \& Ganelin, 2013). Other studies have established that interventions focusing on care coordination are not able to reduce utilization (Finkelstein, 2020), and current ED reduction programs in the literature focus on establishing patients in primary care (Raven, 2016). In community paramedicine programs, paramedics collaborate with interdisciplinary partners to address the needs of patients outside of traditional health care settings. The Gainesville Community Resource Paramedic Program (Gainesville CRP) was implemented in 2017 to provide an intervention to address social determinants that primary care providers (PCP) cannot address.

Methods. A Student's t-test for paired samples was used to compare total ED visits as well as ED visits with and without hospital admission six months before and after program enrollment. Pearson's correlation between final change in total ED visits and total PCP visits for pre-CRP, post-CRP, and full-CRP were calculated to determine if there was evidence for bias in utilization patterns.

Results. Among program participants $(n=53)$, significant reductions were observed among total (mean $=$ $2.94, p<0.001$ ), without admission (mean $=1.30, p=0.006$ ), and with admission (mean $=1.64, p=$ 0.002). Significant reductions were also found among selected demographics and morbidities. Reductions in total ED visits were not associated with visits to a PCP before, after, or throughout.

Conclusions. Gainesville CRP was able to reduce ED visits independent of PCPs indicating that the approach could inform other ED diversion programs.

\section{Contributions To The Literature}

- Provides evidence that addressing the social determinants of health as a primary goal is a key component to the success of Emergency Department diversion programs

- This is the only known community paramedicine program in the literature that focuses on social determinants

- Showcases a methodology for measuring effectiveness of Emergency Department diversion programs that accounts for "reversion to the mean" in retrospective evaluations where without randomization or a control

- Showcases a model design for other communities that wish to develop these programs

- Contributes a tangible solution for communities for improving health equity

\section{Background}

The U.S. healthcare system has consistently struggled with inefficiencies in Emergency Department (ED) usage (Enard \& Ganelin, 2013). Frequent ED users comprised " $4.5 \%$ to $8 \%$ of all ED patients but account for $21 \%$ to $28 \%$ of all visits" (LaCalle, 2010). Furthermore, $13 \%$ to $27 \%$ of ED visits in the United States 
could be managed in primary care settings, with an estimated savings of $\$ 4.4$ billion annually (Weinick, et al., 2010). Many of these individuals suffer from preventable health issues that could be resolved through early intervention and health education (Dowd, et al., 2014).

ED visit reduction programs vary widely in strategy, provider composition, and effectiveness (Raven, 2016). Recent evidence has indicated that programs focused on providing post-acute care coordination for frequent utilizers of ED services may not be effective in reducing overall hospital utilization (Finkelstein, 2020). In order to identify effective methods for reducing visits from frequent ED users, a local community paramedicine program focusing on providing support for social determinants was selected to evaluate and study. While community paramedicine programs have been evaluated in the past (Bigham, 2013), very few programs have focused their intervention specifically on social determinants. By evaluating a locally supported, currently functioning, and unique adaptation of ED visit reduction programs, this study has the potential to help inform health care delivery improvement efforts throughout the U.S.

\section{Community Paramedicine}

As communities have searched for innovative strategies to break the cycle of poor health and high demand on community services, the community paramedicine (CP) model has emerged nationwide as a promising evidence-based approach (O'Meara 2014). The CP model seeks to improve health outcomes, system costs, patient quality, and utilization efficiency within established emergency medical services (EMS) infrastructure (Gregg, 2019). In CP programs, Fire Rescue or EMS departments utilize front-line paramedics in collaboration with interdisciplinary partners such as physicians and social workers to address the needs of these vulnerable patients with complex medical and social needs. The CP model has been successful outside the U.S. for some time (Snooks, 1998) and has been recently adapted to address specific inequity and inefficiency issues particular to the U.S. healthcare system (lezzoni, 2016). $\mathrm{CP}$ and other types of mobile integrated health care $(\mathrm{MIH})$ programs have been effective in reducing frequent ED utilization (Nejtek, 2017), improving chronic disease measures (Bennett, 2018), generating savings by partnering with payer and provider networks (Roeper, 2018), and assisting primary care physicians with care coordination (Chellappa, 2018).

\section{The Gainesville Community Resource Paramedic Program}

In 2017, over 189 Gainesville residents called 911 more than five times in one year, and thirty-nine of those patients called 911 more than ten times in one calendar year. In order to address these needs, the Gainesville Community Resource Paramedic Program (Gainesville CRP) was established in 2017. Gainesville CRP is a collaboration between University of Florida Health (UF-Health) and Gainesville Fire Rescue (GFR) designed to support patients with complex health and social support needs in Alachua County and reduce ED visits at UF-Health Shands Hospital (Shands).

The Gainesville Community Resource Paramedicine (CRP) program was created by Gainesville Fire Rescue in search of a new paradigm for delivering emergency medical services. In this model, a 
paramedic and resource coordinator utilize a non-fire apparatus and visit patients in their homes following a referral for frequent use of ED services. The focus of the program is to address social determinants of health and resolve underlying issues of frequent ED utilization. Gainesville CRP personnel comprehensively evaluate and directly address social needs of patients including food security, transportation, employment, and disability access. Furthermore, Gainesville CRP personnel communicate with healthcare providers in real-time to ensure patients are adhering to their medication and treatment plans, convey details of their complete social picture, while also conducting detailed wellness checks on patients of particularly high-risk. These interactions last for a minimum of six months and services are provided directly in a patient's home. Gainesville CRP coordinates with and is overseen by UF-Health family practice physicians.

While CP and ED visit reduction programs are not new (Thompson, 1991), the use of CP to connect patients to existing community resources not traditionally provided by health systems while also working alongside an established PCP is unique (Siddle, 2018). Most other previously studied ED reduction programs offered only case management and care coordination until the patient could be established in primary care. Previous research has indicated that frequent ED users are "as likely as infrequent users to have a usual source of care" (Cunningham, 2017) and that other research has shown that these patients "do not believe" that PCPs can provide non-medical but health related support (Enard, 2017). Gainesville CRP is designed to provide a resource focused and community embedded intervention that can improve social determinants of health that PCPs or acute care facilities cannot address. This unique approach represents a significantly more involved intervention than many other "complex care" or "hot-spotting programs" programs (lovan, 2020) currently in the literature.

The goal of this coordinated effort is to reduce avoidable hospital visits and 911 calls as well as improve the quality of life of those served by the program. After its first two years of implementation, the Gainesville CRP program has become a nexus in Alachua County connecting patients to social and medical services while fostering relationships ensuring a safety net for self-management of chronic disease (Caplan, 2019).

\section{Literature Review}

In order to evaluate the initial implementation of Gainesville CRP, surveying existing literature is necessary to place the current program in context. While Gainesville CRP is founded on a CP model, the focus on social determinants and community support services places Gainesville CRP as a unique adaptation of the CP model. Identifying the ways in which this kind of intervention is similar or different to existing studies will provide context for the effective components of the program. Also, since the program has already been implemented and does not have a randomization process or a prospective evaluation strategy, understanding the evidence from the pilot in context of study design limitations is needed. Finally, this study should setup a more thorough randomized control trial (RCT) or prospective cohort (PC) in the future that reflects the needs of current literature in the field.

\section{Misconceptions in Emergency Department Utilization}


There is a common assumption that frequent use of the Emergency Department is the result of indigent or underinsured patients who wish to access free sources of care in emergency settings rather than access care through a primary care provider. This assumption also holds that many of these visits are low-acuity, non-emergent, and would be most appropriately handled in a primary or secondary care office. The framework in Figure 1 illustrates the nature of this assumption:

In this model, indigent patients with a history of cost avoidance in health care consumption are subsequently not established in primary care. When need for care is presented, this pattern will lead to ED visits out of concern for cost and a lack of other available options. This is contrasted with non-indigent patients that are insured, established in primary care, and expect to participate in cost sharing as a responsible consumer of health care services. When need for care arises, they will consult with a primary care physician rather than visit an ED. Under this framework, the interventions that would seek to alleviate the inappropriate use of EDs would seek to establish indigent patients in primary care so that future care needs could be addressed in more appropriate spaces.

Unfortunately, this framework does not adequately explain the demographics of frequent ED users and their reasons for visiting the ED. Frequent ED users tend to have established PCPs (Zhou, 2013), health insurance coverage (Zhou, 2017), utilize care at higher rates in both ED and non-ED settings including PCP visits (Giannochous 2019), are between the ages of nineteen and forty-four (Giannochous 2019), visit the ED for exacerbations of common chronic diseases (Vinton, 2013), have lower self-reported health status (Vinton, 2013), and utilize the ED for services that PCPs are unable to deliver (LeCalle, 2010). This indicates that the frequent ED user is not primarily utilizing out of a lack of establishment in a PCP, but instead has a higher level of complexity in chronic disease that requires extra care outside of services that a PCP can offer. Similarly, food insecurity was identified in a number of studies as a significant predictor of frequent ED use (Giannochous, 2019) suggesting that social determinants may play an outsized role in predicting exacerbated health needs requiring an ED visit for this population. From this perspective, interventions that focus on case management or care coordination will not be able to address the underlying lack of services that would be necessary to reduce utilization. These approaches would only connect patients to services that they already may be utilizing at higher levels. Instead, interventions would need to identify the components of chronic disease care that are not being provided to patients as well as the social determinants that are not being addressed by existing care services.

In a survey of frequent Emergency Department utilizers, LeCalle writes:

"First, the data from these studies challenge the common assumption that frequent ED use is a problem of uninsured, ethnic minority patients inappropriately seeking basic primary care in the ED... Furthermore, much of the frequent ED use is not for primary care. Although some patients certainly use the ED multiple times for low-acuity complaints, the consensus in the literature is that frequent users often are sick patients with chronic illness associated with high admission rates and high mortality." (2010)

While studies like this one have established the discrepancy between assumptions and reality in the context of ED use, almost all programs that sought to alleviate frequent ED utilization focused on care 
coordination or case management (Raven, 2016). In a more recent review, studies that only included care coordination showed none or mixed results (Iovan, 2020). In a scoping review of studies in CP, only four of the eight identified studies included social support services outside of healthcare and none were specifically focused on providing care that meaningfully addressed social determinants (Gregg, 2019). While comprehensive improvements to primary care delivery emerging alongside value-based payment programs may help in improving frequent $\mathrm{ED}$ usage, $\mathrm{CP}$ has the potential to help address upstream needs while integrating seamlessly into current health care delivery.

\section{The Need for More Robust Methodology}

When evaluating the current data from the pilot CRP program, a method needed to be developed that can account for ED use independent of PCP visits before and after enrollment. Similarly, this method must be able to identify evidence that can also account for possible "reversion to the mean" that occurs as a result of a natural drop in overall utilization. Of the studies that utilized observational data for CP interventions, no studies attempted to account for either of these contexts.

There is also a significant need for CP studies that utilize a PC or RCT design to be published in order to advance the implementation of CP throughout the U.S. Recent evidence from the Camden Coalition (Finkelstien, 2019), indicates that when random assignment is used to control for biases in evaluating ED diversion programs, a significant "reversion to the mean" is observed. In a review of ED reduction programs, authors noted that "methodological and study design weaknesses -especially regression to the mean- were widespread and call into question reported positive findings" (lovan, 2020). Based on the existing literature, observational; study design methods need to be developed that can account for the above limitations and provide robust evidence for future PC or RCT studies.

\section{Methods}

In order to evaluate the impact of the CRP program, patient ED visits at Shands were compared six months before and six months after enrollment in the program $(N=65)$ from March 2017 to October 2019. Among patients with more than one ED visit $(N=53)$, a Student's t-test for paired samples was used to compare each patients pre and post enrollment total ED visits, "treat and release" (T\&R) visits, and visits with hospital admission. This test provides evidence on the possible effect of CRP to reduce ED visits among patients before and after enrollment.

\section{Assessing Possible Biases in the Observational Study}

In order to understand possible confounders and biases, PCP visits were also collected six months before and six months after CRP enrollment. By collecting PCP visits, the change in ED utilization before and after CRP enrollment can be compared with PCP visits in order to address three potential null hypotheses that would provide evidence against CRP having an effect on ED utilization drops. Those three null hypotheses are: 
1. CRP has no effect and overall utilization (PCP and ED visits) drops naturally. This hypothesis would state that patients had abnormally high utilization when referred and would eventually "revert to the mean" with or without CRP. Any impact would be an artifact of established trends in utilization spikes that resolve naturally (Barnett, 2005). This hypothesis partially explains the result from the Camden study (Finkelstein, 2019). If PCP visits are used as a proxy for overall utilization, patients that naturally reduce utilization would experience both a drop in PCP and ED visits after CRP. Evidence of this hypothesis would come from a positive correlation between ED visit drop and PCP visits after CRP.

2. CRP's effect is mediated by PCP visits. This hypothesis would state that access to a PCP accounts for the drop in ED visits and that in the Post-CRP six months, PCP visits replace ED visits. This is based on the theory that patients are going to the ED instead of a PCP out of convenience, preference, or cost and PCP establishment relieves frequent ED use. Evidence of this hypothesis would come from a negative correlation between ED visit drop and PCP visit after CRP.

3. CRP's effect is confounded by PCP visits. This hypothesis would state that patients that were already working with primary care pre-CRP experienced an ED visit drop. Since many patients had PCP visits before and after, it is possible that CRP had no effect and that patients with more PCP visits through the entire twelve months (or six months pre) experienced ED visit drop from the effect of a PCP. Evidence of this hypothesis would come from a negative correlation between ED visit drop and PCP visits either before CRP or for the full 6 months of data.

While this is designed to be a retrospective observational study of a pilot program without a control group or randomization, the use of PCP visits to gather evidence of possible confounders and biases can address some of the significant issues present in the evidence of these types of programs (lovan, 2020). Pearson's product moment correlation between final change in total ED visits and total PCP visits for preCRP, post-CRP, and full-CRP was calculated to determine if there was evidence to support any of the three null hypotheses above.

Institutional Review Board oversight was provided by the University of Florida under IRB- Exempt (IRB201901820). While external funding sources were not used to study the program, the City of Gainesville has received a National Science Foundation planning grant to explore ways to expand the program staring in Fall of 2020 after this study was completed. Reporting guidelines were provided using the Strengthening the Reporting of Observation Studies in Epidemiology checklist.

\section{Results}

Statistically significant average reductions were observed among total (mean difference $=2.94, \mathrm{p}<$ 0.001 ), treat and release (mean difference $=1.30, p=0.006$ ), and hospital admission ED visits (mean difference $=1.64, p=0.002$ ). These results shown in Figure 2 and referenced in Table 1. Significant reductions were also found among Female, non-Hispanic Black, patients 65 and under, patients with hypertension, and patients with Type II diabetes. These are referenced in Table 2. Average reductions in 
total ED visits were not associated with visits to a PCP before CRP $(r=-0.188, p=0.852)$, after CRP $(r=$ $-0.164, p=0.565)$, and 12 -month total $(r=-0.144, p=0.557)$. These results are referenced in Table 3 .

\section{Discussion}

The observed drop in all types of ED visits before and after enrollment for all demographics and morbidities provides evidence that the unique aspects of Gainesville CRP's intervention had a strong, statistically significant effect. Furthermore, the lack of statistically significant correlations between PCP visits and ED visit drop also indicates that CRP participation had an independent effect on ED utilization outside of patient PCP visits before or after. This also provides evidence that ED visit reductions resulting from CRP enrollment may not be biased through "regression to the mean" as ED visit drop did not have a statistically significant correlation with visits to a PCP during the 12 months of patient data. Since the Gainesville CRP intervention is focused on providing supportive services designed specifically to address the social determinants that are unable to be addressed within a PCP visit, these results are consistent with the model of the program. This also provides evidence that other ED diversion programs that utilize significant resources for the goal of reducing avoidable ED visits should employ a similar approach of supplementing PCP care rather than diverting to PCP care. Therefore, the unique aspects of the Gainesville CRP intervention are informative to future efforts in the area of ED diversion.

\section{Limitations}

This was an observational study on a small retrospective cohort and the conclusion that ED diversion programs focusing on social determinants instead of PCP referral will need to be validated with a RCT or PC design that can account for other unknown biases. Because the results from the Camden Coalition did not confirm observational and anecdotal data from other studies, conclusions about CRP will need to be tested with a similar robust study design in the immediate future. These results do however provide the foundational evidence that such a study has the potential to identify positive results with the Gainesville CRP approach.

\section{Conclusion}

The Gainesville Community Resource Paramedic program was designed to address social determinants for frequent ED users outside of the scope of primary care providers with a community paramedicine approach. Gainesville CRP was able to significantly reduce ED visits for patients for six months after enrollment. Because this reduction was not associated with PCP visits before or after enrollment, there is evidence that the unique approach of the program has potential to inform ED diversion programs and provide the basis for a more robust study.

\section{Abbreviations}

ED - Emergency Department 
CRP - The Gainesville Community Resource Paramedic Program

PCP - Primary care providers

$\mathrm{CP}$ - Community Paramedicine

UF-Health - University of Florida Health

GFR - Gainesville Fire Rescue

Shands - UF-Health Shands Hospital

RCT - Randomized control trial

PC - Prospective Cohort

\section{Declarations}

\section{Ethics approval and consent to participate}

Patient data was acquired through a retrospective chart review provided by UF-Health Shands and accessed through Institutional Review Board oversight at the University of Florida (IRB- Exempt IRB201901820).

\section{Consent for publication}

Consent for publication was provided by UF-Health Shands and through Institutional Review Board oversight at the University of Florida (IRB- Exempt IRB201901820).

\section{Availability of data and materials}

The datasets analyzed during the current study are not publicly available due to privacy guidelines related to the dissemination of patient data but are available from the corresponding author on reasonable request.

\section{Competing interests}

The authors declare that they have no competing interests to disclose.

\section{Funding}

The authors declare that they have no funding sources to disclose. The City of Gainesville has received a National Science Foundation planning grant to explore ways to expand the program staring in Fall of 2020 after this study was completed.

\section{Author Contributions and Information}


Andrew Cistola MPH is a PhD student in the Health Services Research program at the University of Florida and a Health Data Analyst at the Florida Department of Health.

Ariella N. Bak EMTP functions as the program director of the Gainesville Fire Rescue Community Paramedic Program and is a part-time student in the Masters of Health Administration at Florida International University.

Laura Guyer PhD, MEd, RDN provides leadership to the undergraduate Health Disparities in Society minor and publishes the Alachua County Community Health and Social Services Resource Guide annually.

Austin Reed MD is a fellow in the Department of Emergency Medicine at the University of Florida Health Shands.

Ben Rooks MA is a research coordinator in the Department of Community Health and Family Medicine.

Lisa Chacko MD, MPH volunteered as the medical director of the Gainesville Community Resource Paramedic Program and recently was a primary care physician with the Department of Community Health and Family Medicine. Lisa Chacko also advises the City of Gainesville on efforts for health improvement for their citizens.

Andrew S. Cistola, Ariella N. Bak, Laura Guyer, and Lisa Chacko also serve on the Alachua County Safety Net Collaborative.

\section{Acknowledgements}

This paper and the operational efforts behind the CRP Program would not have been possible without the exceptional support of Gainesville Fire Rescue's Chief of Training David Roberto Sutton and Chief of Operations Joseph Shawn Hillhouse. Pioneering the Gainesville community paramedicine concept, David Sutton is the visionary behind the operation. His enthusiasm for innovation and vision casting proves his strong leadership skills and dedication to service. Chief Sutton's infectious positivity inspires those around him to strive for more. From being an instructor to co-worker, it has been nothing short of an honor to work beside Chief Sutton in bringing his vision to life. Chief Hillhouse embodies the epitome of service leadership. He continuously pours belief and empowers his co-works and employees while guiding them to showcase their passions. Chief Hillhouse prioritizes the needs of his employees and supports any effort to combine passion with the vision for the fire department welcoming innovation and creativity which is typically rare in a traditional fire service. In regards to the creation of the CRP Program, Chief Hillhouse caught wind of a vision and works daily to ensure the program is succeeding in every aspect guided by his oversight. Chief Hillhouse does not perceive obstacles rather embraces opportunity and calls upon a team mentality to push towards success. I am forever humbled and honored to work alongside Chief Hillhouse where I am given the opportunity daily to innovate in the name of helping people. 
The authors would also like to acknowledge the numerous GFR firefighters, paramedics and Resource Technicians who are the reason the CRP Program continues to be successful and make a significant impact on the Gainesville community. Kamelia Klejc and Payton Campbell have stepped into uncharted roles serving as the primary Resource Technicians for the CRP Program managing cohorts of patients and connecting every medical and socially based need. These women have gone above and beyond their duties in times of hardship in the face of the COVID-19 pandemic to ensure relief was brought to each and every patient. Kamelia and Payton show up daily with an attitude of service unphased by any obstacles that may arise. It has been such an honor to watch the growth process.

\section{References}

1. Barnett, A. G., van der Pols, J. C., \& Dobson, A. J. (2005). Regression to the mean: what it is and how to deal with it. International Journal of Epidemiology, 34(1), 215-220. https://doi.org/10.1093/ije/dyh299

2. Bennett, K. J., Yuen, M. W., \& Merrell, M. A. (2018). Community paramedicine applied in a rural community. The Journal of Rural Health, 34 Suppl 1, s39-s47. https://doi.org/10.1111/jrh.12233

3. Bigham, B. L., Kennedy, S. M., Drennan, I., \& Morrison, L. J. (2013). Expanding paramedic scope of practice in the community: a systematic review of the literature. Prehospital Emergency Care, 17(3), 361-372. https://doi.org/10.3109/10903127.2013.792890

4. Caplan, Andrew (2019). Program aims to reduce 911 calls, ER visits. Gainesville Sun. Retrieved August 29, 2020, from https://www.gainesville.com/news/20190525/program-aims-to-reduce-911calls-er-visits

5. Chellappa, D. K., DeCherrie, L. V., Escobar, C., Gregoriou, D., \& Munjal, K. G. (2018). Supporting the oncall primary care physician with community paramedicine. Internal Medicine Journal, 48(10), 12611264. https://doi.org/10.1111/imj.14049

6. Dowd B, Karmarker M, Swenson T, et al. Emergency department utilization as a measure of physician performance. Am J Med Qual 2014;29(2):135-43. http://ajm.sagepub.com/content/29/2/135.long. Accessed June 16, 2016.

7. Enard, K. R., \& Ganelin, D. M. (2017). Exploring the Value Proposition of Primary Care for Safety-Net Patients Who Utilize Emergency Departments to Address Unmet Needs. Journal of Primary Care \& Community Health, 8(4), 285-293. https://doi.org/10.1177/2150131917721652

8. Enard KR, Ganelin DM. Reducing preventable emergency department utilization and costs by using community health workers as patient navigators. J Healthc Manag 2013;58(6);412-28. http://www.ncbi.nlm.nih.gov/pmc/articles/PMC4142498/. Accessed June 16, 2016.

9. Finkelstein, A., Zhou, A., Taubman, S., \& Doyle, J. (2020). Health Care Hotspotting - A Randomized, Controlled Trial. The New England Journal of Medicine, 382(2), 152-162. https://doi.org/10.1056/NEJMsa1906848

10. Gregg, A., Tutek, J., Leatherwood, M. D., Crawford, W., Friend, R., Crowther, M., \& McKinney, R. (2019). Systematic review of community paramedicine and EMS mobile integrated health care interventions 
in the united states. Population Health Management, 22(3), 213-222.

https://doi.org/10.1089/pop.2018.0114

11. lezzoni, L. I., Dorner, S. C., \& Ajayi, T. (2016). Community paramedicine - addressing questions as programs expand. The New England Journal of Medicine, 374(12), 1107-1109. https://doi.org/10.1056/NEJMp1516100

12. Iovan, S., Lantz, P. M., Allan, K., \& Abir, M. (2020). Interventions to Decrease Use in Prehospital and Emergency Care Settings Among Super-Utilizers in the United States: A Systematic Review. Medical Care Research and Review, 77(2), 99-111. https://doi.org/10.1177/1077558719845722

13. LaCalle, E., \& Rabin, E. (2010). Frequent users of emergency departments: the myths, the data, and the policy implications. Annals of Emergency Medicine, 56(1), 42-48.

https://doi.org/10.1016/j.annemergmed.2010.01.032

14. Nejtek, V. A., Aryal, S., Talari, D., Wang, H., \& O’Neill, L. (2017). A pilot mobile integrated healthcare program for frequent utilizers of emergency department services. The American Journal of Emergency Medicine, 35(11), 1702-1705. https://doi.org/10.1016/j.ajem.2017.04.061

15. O'Meara, P. (2014). Community paramedics: a scoping review of their emergence and potential impact. International Paramedic Practice, 4(1), 5-12. https://doi.org/10.12968/ippr.2014.4.1.5

16. Raven, M. C., Kushel, M., Ko, M. J., Penko, J., \& Bindman, A. B. (2016). The effectiveness of emergency department visit reduction programs: A systematic review. Annals of Emergency Medicine, 68(4), 467-483.e15. https://doi.org/10.1016/j.annemergmed.2016.04.015

17. Roeper, B., Mocko, J., O’Connor, L. M., Zhou, J., Castillo, D., \& Beck, E. H. (2018). Mobile Integrated Healthcare Intervention and Impact Analysis with a Medicare Advantage Population. Population Health Management, 21(5), 349-356. https://doi.org/10.1089/pop.2017.0130

18. Siddle, J., Pang, P. S., Weaver, C., Weinstein, E., O’Donnell, D., Arkins, T. P., Miramonti, C., \& MIH CORE team. (2018). Mobile integrated health to reduce post-discharge acute care visits: A pilot study. The American Journal of Emergency Medicine, 36(5), 843-845.

https://doi.org/10.1016/j.ajem.2017.12.064

19. Snooks, H., Wrigley, H., George, S., Thomas, E., Smith, H., \& Glasper, A. (1998). Appropriateness of use of emergency ambulances. Journal of Accident \& Emergency Medicine, 15(4), 212-215. https://doi.org/10.1136/emj.15.4.212

20. Thompson, R. H., \& Wolford, R. W. (1991). Development and Evaluation of Criteria Allowing Paramedics to Treat and Release Patients Presenting with Hypoglycemia: A Retrospective Study. Prehospital and Disaster Medicine, 6(3), 309-313. https://doi.org/10.1017/S1049023X00038747

21. Weinick RM, Burns RM, Mehrotra A. How many emergency department visits could be managed at urgent care centers and retail clinics? Health Aff 2010;29(9):1630-6.

http://www.ncbi.nlm.nih.gov/pmc/articles/PMC3412873/. Accessed June 16, 2016.

22. Zhou, R. A., Baicker, K., Taubman, S., \& Finkelstein, A. N. (2017). The Uninsured Do Not Use The Emergency Department More-They Use Other Care Less. Health Affairs (Project Hope), 36(12), 21152122. https://doi.org/10.1377/hlthaff.2017.0218 


\section{Tables}

Table 1. Patient Visits to UF-Health Shands Emergency Department 6-months Before and After Gainesville CRP Enrollment*

\begin{tabular}{|llllll|}
\hline & Pre-CRP & Post-CRP & Mean Difference & SD & P** \\
\hline Total ED Visits & 361 & 205 & 2.94 & 5.66 & 0.000 \\
\hline Treat and Release & 191 & 122 & 1.30 & 3.27 & 0.006 \\
\hline Visit with Hospital Admission & 170 & 83 & 1.64 & 3.64 & 0.002 \\
\hline Notes: *Patients with one recorded ED visit were included $(n=53)$ & & \\
\hline$* *$ A Student's t-Test for paired samples to test statistical significance & & \\
\hline
\end{tabular}

Table 2. Patient Visits to UF-Health Shands Emergency Department 6-months Before and After Gainesville CRP Enrollment by Patient Demographics*

\begin{tabular}{|c|c|c|c|}
\hline & Demographic & $\mathrm{n}$ & $P * \star$ \\
\hline \multirow[t]{2}{*}{ Gender } & Female & 34 & 0.008 \\
\hline & Male & 19 & 0.024 \\
\hline \multirow[t]{2}{*}{ Race* } & Black & 30 & 0.05 \\
\hline & White & 22 & 0.026 \\
\hline \multirow[t]{2}{*}{ Age } & Over 65 years & 24 & 0.005 \\
\hline & 65 years and under & 29 & 0.038 \\
\hline \multirow[t]{4}{*}{ Diagnoses Present at Visit } & Congestive Heart Failure & 22 & 0.030 \\
\hline & Type II Diabetes & 34 & 0.012 \\
\hline & Chronic Obstructive Pulmonary Disease & 17 & 0.005 \\
\hline & Hypertension & 35 & 0.003 \\
\hline \multicolumn{4}{|c|}{ Notes: *Patients with one recorded ED visit were included $(n=53)$} \\
\hline \multicolumn{4}{|c|}{ **A Student's t-Test for paired samples to test statistical significance } \\
\hline \multicolumn{4}{|c|}{$\begin{array}{l}\star * \star \text { Does not include patients that reported Hispanic ethnicity without race }(n=1) \text {. All other patients } \\
\text { reported non-Hispanic. }\end{array}$} \\
\hline
\end{tabular}


Table 3. Average Reduction in UF-Health Shands Emergency Department Visits and Count of Visits to Primary Care Provider during Gainesville CRP Enrollment*

\begin{tabular}{|lccc|}
\hline \multicolumn{4}{|c|}{$\begin{array}{l}\text { Mean } \\
\text { ED Correlation with } \\
\text { ED Visit Reduction }\end{array}$} \\
\hline ED Visit Reduction by Patient & 2.94 & \\
\hline PCP Visit Count by Patient & & \\
\hline Full CRP & 11.45 & -0.188 & 0.852 \\
\hline Before CRP & 6.32 & -0.164 & 0.565 \\
\hline After CRP & 5.13 & -0.144 & 0.557 \\
\hline Notes: *Patients with one recorded ED visit were included $(n=53)$ \\
\hline **Pearson's correlation used to test statistical significance \\
\hline
\end{tabular}

\section{Figures}

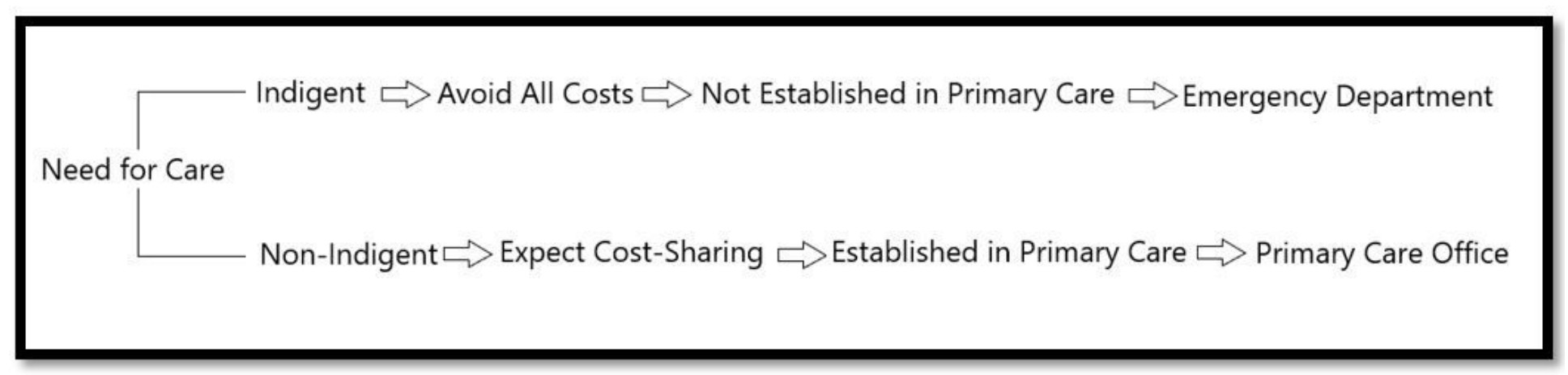

Figure 1

Assumptions in Emergency Department Utilization 


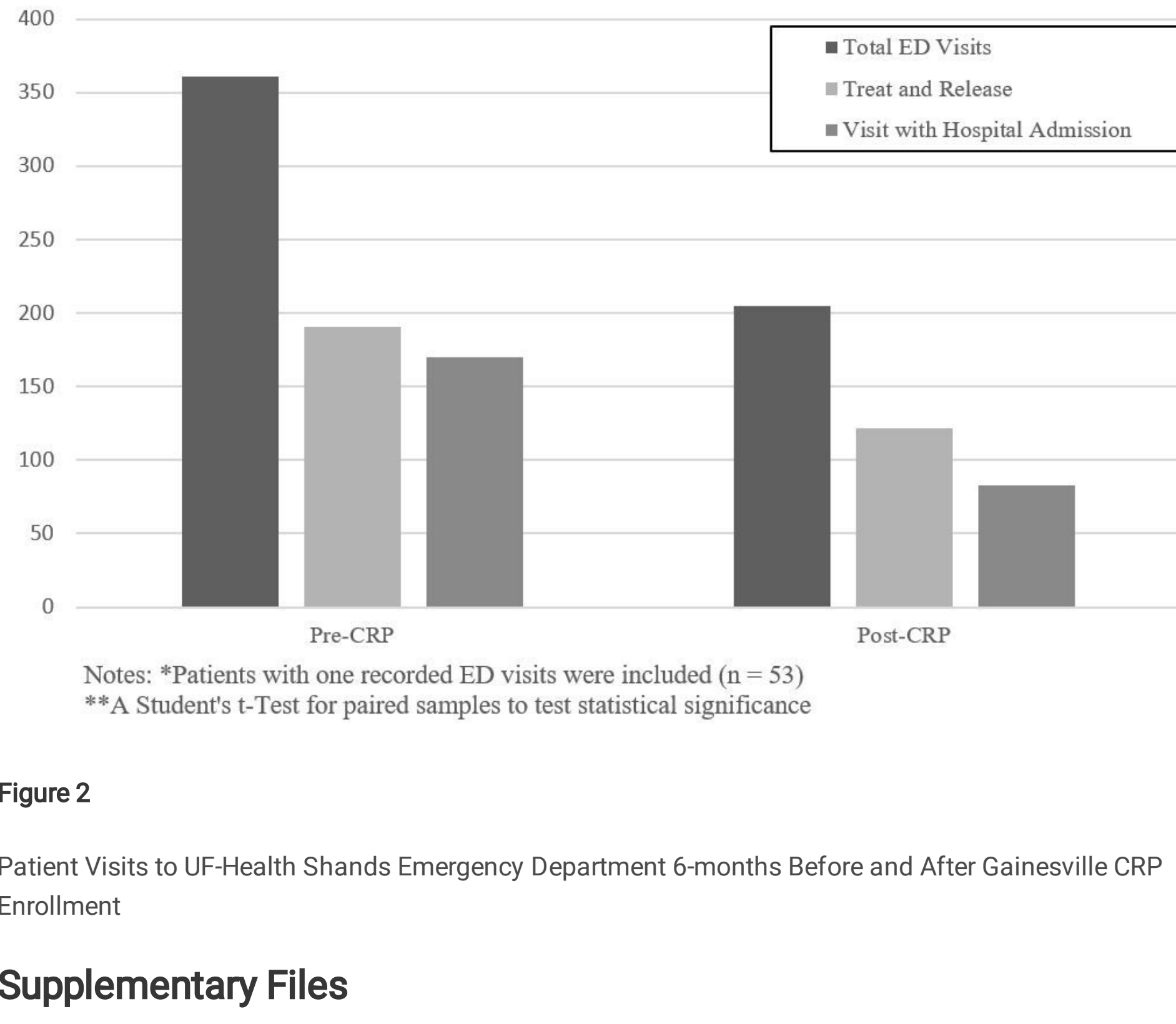

This is a list of supplementary files associated with this preprint. Click to download.

- ReportingStandards.docx 